\title{
Essais
}

Revue interdisciplinaire d'Humanités

\section{Protestation et résistance. Du critère de distinction}

\section{Wolfgang Kraushaar}

Traducteur : Gaëlle Guicheney

\section{(2) OpenEdition}

Journals

Édition électronique

URL : http://journals.openedition.org/essais/5923

DOI : 10.4000/essais.5923

ISSN : 2276-0970

Éditeur

École doctorale Montaigne Humanités

Édition imprimée

Date de publication : 15 janvier 2015

Pagination : 164-178

ISBN : 978-2-9544269-4-5

ISSN : $2417-4211$

\section{Référence électronique}

Wolfgang Kraushaar, «Protestation et résistance. Du critère de distinction », Essais [En ligne], 6 | 2015, mis en ligne le 24 novembre 2020, consulté le 04 décembre 2020. URL : http://

journals.openedition.org/essais/5923; DOI : https://doi.org/10.4000/essais.5923

Ce document a été généré automatiquement le 4 décembre 2020.

Essais 


\title{
Protestation et résistance. $\mathrm{Du}$ critère de distinction
}

\author{
Wolfgang Kraushaar \\ Traduction : Gaëlle Guicheney
}

1 Lorsque l'on se penche sur des catégories telles que la contestation, la protestation et la résistance ${ }^{1}$, il faut être bien conscient que l'on s'expose à des malentendus. Opposer ces concepts et chercher à les définir dans une approche contrastive suppose d'établir au préalable le contexte politique et social dans lequel ils s'inscrivent. Les réflexions suivantes ne font pas référence à un État totalitaire, comme l'État national-socialiste ou le communisme soviétique, qui n'ont, de toute façon, toléré aucune forme de contestation. Elles se réfèrent à un État constitutionnel démocratique ayant mis en place sa propre forme de séparation des pouvoirs. Ces réflexions s'inscrivent donc dans le contexte d'une démocratie qui, malgré ses lacunes, est apte à fonctionner. Il n'y a guère que dans ce cadre précis, celui d'une démocratie parlementaire, que l'on peut raisonnablement confronter les catégories mentionnées.

2 I. En mai 1968 - à l'apogée des mouvements d'opposition extraparlementaire en République fédérale d'Allemagne - une tribune est parue dans la revue Konkret dans laquelle s'annonçait une sorte de tournant politique. En voici les premières phrases: «"Protester, c'est dire : telle et telle chose ne me conviennent pas. Résister, c'est veiller à ce que les choses qui ne me conviennent pas ne se reproduisent plus. Protester, c'est dire: je me retire. Résister, c'est faire en sorte que tous les autres se retirent également." C'est à peu près en ces termes qu'un Noir du Black Power s'est exprimé lors du Meeting international contre la guerre du Vietnam en février dernier à Berlin. $»^{2}$

Qui était ce conférencier noir auquel la journaliste faisait référence, et qu'avait-il dit véritablement? Il s'agissait d'un représentant du Black Power, mouvement qui était en train de remplacer celui en faveur des droits civiques alors en perte vitesse. Cet homme s'appelait Dale A. Smith. Il s'était fixé pour objectif d'établir la différence qui sépare un mouvement de protestation d'un mouvement de résistance: «Protester, c'est se prononcer contre quelque chose, c'est faire savoir qu'on n'apprécie pas certaines actions perpétrées par un autre. Protester est un acte intellectuel... Protester veut dire 
jouer. On participe à une manifestation, on écoute les discours, on hisse des banderoles, puis on rentre chez soi pour se regarder passer à la télévision. Il existe beaucoup de jeux pour jouer à la protestation. $»^{3}$

4 Et, par opposition: "Résister, c'est dire non sans fournir davantage d'explication... Résister, c'est poser les principes de la vie telle que tu l'entends, contre les principes de la vie telle que eux l'entendent, et faire tout ce qui est nécessaire pour voir leur définition entièrement anéantie. $»^{4}$

5 Et à la fin de sa présentation, il résume une dernière fois : " Protester signifie abhorrer l'inhumanité d'une personne. Résister, c'est réprimer l'inhumanité et faire triompher l'humanité. On ne proteste pas contre un meurtre - on s'empare du meurtrier pour le traiter comme il le mérite.»

6 Pour tous les auditeurs présents ce jour-là dans l'Auditorium maximum de l'Université technique de Berlin, la personne visée ne faisait aucun doute. Il était question du président des États-Unis alors en place, Lyndon B. Johnson, et du corps militaire responsable des interventions au Vietnam. Le discours résonna comme un appel au meurtre - à traiter le meurtrier comme il le méritait prétendument.

7 La personne qui fut marquée par ce discours et qui s'en est fait l'écho dans Konkret n'est autre qu'Ulrike Meinhof, la future co-fondatrice de la RAF 監Rote Armee Fraktion臨, la Fraction Armée Rouge. Elle cherchait à montrer où l'engrenage qui s'était enclenché lors des violentes réactions suscitées par l'attentat contre l'étudiant Rudi Dutschke en avril 1968 allait mener.

8 Ce que l'on peut toutefois remarquer dans sa tribune, c'est qu'elle ne se prononce pas en faveur d'un simple recours à la violence. Au contraire. "La contre-violence », ainsi définit-elle les débordements militants, «menace de devenir violence à partir du moment où la brutalité policière détermine les règles qui encadrent les actions, lorsqu'une une fureur impuissante remplace une rationalité supérieure, lorsque l'on répond à l'intervention paramilitaire de la police par des moyens paramilitaires. "

Un raisonnement auquel Meinhof elle-même n'a visiblement pas longtemps adhéré. Car les pratiques de la RAF peuvent être considérées comme l'entière négation de cette critique.

II. Les nouvelles formes d'actions qui ont été pratiquées au début du mouvement de 68 et qui, dans l'opinion publique, provoquèrent un rejet voire de furieuses contreréactions, ont très vite été estampillées d'une étiquette censée restreindre et minimiser leurs potentiels provocateurs: on a considéré qu'elles relevaient d'une stratégie de l'« infraction restreinte» [ « begrenzte Regelverletzung »]. Dans le cas des sit-in et des occupations de locaux, il était clair que ces actions ne relevaient que d'infractions au règlement intérieur ou d'autres délits plutôt anodins. Il ne s'agissait pas de conséquences collatérales involontaires, mais d'actions délibérément assumées. Dans de nombreux cas, l'attrait spécifique de l'action résidait justement dans le fait qu'elle ne respectait ni l'ordre établi ni la loi ${ }^{5}$.

11 Du point de vue des acteurs, le problème consistait avant tout à prévenir les débordements et à réduire au minimum les infractions commises lors des actions. Car ceux-ci ne cherchaient pas à montrer qu'une loi avait été violée, mais plutôt à souligner le caractère symbolique d'une transgression commise contre des règles ou des lois en vigueur. Il s'agissait de signaler clairement à l'opinion publique qu'eux-mêmes 
prenaient au sérieux les infractions aux règles. Mais en même temps, il ne devait faire aucun doute que l'objectif visé ne résidait pas dans l'exécution d'actions illégales.

12 Au cours d'un sit-in organisé dans l'Auditorium maximum de l'Université libre de Berlin, l'étudiant germaniste Peter Schneider a fait en avril 1967 un discours qui exprime mieux que tout autre témoignage le sens ainsi que les motifs déterminants à l'origine des infractions restreintes. Dans son allocution, qui fut par la suite publiée sous le titre « Nous avons fait des erreurs » [Wir haben Fehler gemacht], il déclare :

Nous avons rendu compte en toute objectivité de la guerre du Vietnam, bien que l'expérience nous ait montré qu'il est possible de livrer les détails les plus inconcevables concernant la politique américaine qui sévit au Vietnam sans qu’à aucun moment l'imagination de nos voisins ne se mette en marche, alors qu'il nous suffit de marcher une fois sur une pelouse malgré l'interdiction pour susciter un franc sentiment d'horreur général et tenace.

Nous avons manifesté de manière tout à fait démocratique contre les lois d'exception [Notstandgesetze], bien que nous ayons vu qu'il est possible d'énumérer tous les rangs du service civil sans réveiller le moindre souvenir, alors qu'il nous suffisait de modifier l'itinéraire d'une manifestation prévu par la police pour tirer le maire et les habitants de la capitale de leur lit.

Nous avons revendiqué dans le calme et dans les règles une réforme de l'Université, bien que nous nous soyons aperçus que nous pouvions exprimer notre désaccord vis-à-vis des structures universitaires autant que nous le voulions, sans que jamais le moindre dossier ne soit ouvert, alors qu'il nous suffit d'aller à l'encontre des règles édictées par le service d'urbanisme pour faire vaciller l'organisation universitaire tout entière.

C'est alors que nous en sommes venus à penser qu'il nous fallait d'abord détruire la pelouse, avant de pouvoir détruire les mensonges colportés sur le Vietnam, qu'il nous fallait d'abord modifier l'itinéraire des manifestations, avant de pouvoir modifier quelque chose dans les lois d'exception, qu'il nous fallait d'abord renverser le règlement intérieur avant de pouvoir renverser l'ordre universitaire.

Ainsi en sommes-nous arrivés à l'idée que l'interdiction de marcher sur la pelouse, l'interdiction de modifier l'itinéraire de la manifestation, l'interdiction de se rassembler sans l'accord du service de l'urbanisme, étaient précisément les interdictions avec lesquelles les dirigeants s'assuraient que l'indignation soulevée par les crimes commis au Vietnam, par les structures universitaires archaïques, reste bien tranquille et sans retombées.

Nous nous sommes alors rendu compte que c'est dans de telles interdictions que se décharge l'indifférence criminelle de toute une nation.

Nous avons donc fini par comprendre que, face à la folie des titres et du décorum qui sévissent dans les universités, face à leurs tribunaux d'exception, face à face aux examens pour lesquels on n'apprend que la peur, face aux séminaires où l'on n'enseigne qu'à compulser des livres, face aux programmes de formation qui systématiquement nous déforment, face à leur objectivité qui ne signifie rien de plus que de la fatigue, face à la diffamation perpétrée à l'encontre de toute émotion par les dirigeants afin de s'octroyer le droit de parler des tortures exercées au Vietnam avec le même calme que s'ils parlaient de la météo, face à un comportement démocratique qui sert à étouffer la démocratie, face au calme et à l'ordre dans lesquels se prélassent les oppresseurs, face à la rationalité mensongère et à la complaisante pauvreté des sentiments - face à tout ce vieux fatras, nous avons fini par comprendre que la façon d'argumenter la plus réaliste pour nous est d'arrêter d'argumenter et de nous asseoir par terre dans ces couloirs. C'est ce que nous allons faire à présent. ${ }^{6}$

13 Déployer une telle argumentation pour ne rien faire d'autre que de s'asseoir par terre, voilà qui est aujourd'hui à peine concevable. 

pour lesquelles une protestation organisée dans le cadre prescrit par les autorités, la police et la Justice était vouée à l'échec. Il dresse le bilan d'un légalisme qui s'était révélé inefficient tout au long de l'histoire de la République fédérale. Appels, requêtes, pétitions, lettres ouvertes - rien n'y avait fait. Les initiatives et tentatives s'étaient succédé ; pourtant, pratiquement rien n'avait abouti à quoi que ce soit. Au bout du compte, les vieux dysfonctionnements ont continué de s'empiler, les réformes se sont accumulées dans un flou artistique de plus en plus total, et un sentiment de frustration s'est profondément ancré chez ceux qui non seulement avaient voulu des changements, mais qui avaient tout fait pour les promouvoir. évidence l'absurdité immanente de toute une série de règles, de principes d'ordre et d'autres lois, et formulé une issue : l'atteinte portée à la norme, la transgression de la légalité, l'infraction restreinte. Même si le contexte à Berlin-Ouest n'était pas comparable à celui des États du Sud aux USA, le discours de Schneider puisait dans l'esprit de Martin Luther King et du mouvement américain des droits civiques. Chez les étudiants de l'Université libre de Berlin, l'idée de désobéissance civile allait trouver un terrain favorable. spécialiste de philosophie sociale de Francfort Jürgen Habermas, qui rendit hommage aux nouvelles formes de protestation. Longtemps mentor du SDS 㗨Sozialistischer Deutscher Studentenbund區 ('Union socialiste allemande des étudiants), il avait exercé par le passé une influence déterminante sur l'image que les activistes universitaires se faisaient d'eux-mêmes, avec son étude intitulée «Du concept de participation politique $»^{7}$, puis sa thèse d'habilitation à diriger des recherches "L'espace public $»^{8}$, avant de se heurter à un violent rejet de la part des étudiants ainsi que de certains collègues $^{9}$ en raison de ses vives critiques lancées contre la conception actionniste de la politique adoptée par Rudi Dutschke et d'autres membres dirigeants du SDS ${ }^{10}$.

L'éloge qu'inspiraient à présent à Habermas les succès produits par ce qu'il appelait "les inventions imaginatives en termes de nouvelles techniques de manifestation " était emballé dans un règlement de compte avec le SDS au sujet de l'image que celui-ci se faisait de lui-même, ainsi que de la stratégie et de la tactique choisies par sa politique d'action. Les thèses qu'il avança le 2 juin 1968 dans le restaurant universitaire de l'Université de Francfort lors d'un congrès réunissant des lycéens et des étudiants organisé par le VDS [Verband deutscher Studentenschaft], l'association des étudiants allemands, furent relayées peu après sous le titre provocateur de «La révolution fictive et ses enfants » dans une édition spéciale du quotidien Frankfurter Rundschau ${ }^{11}$.

18 "Les nouvelles techniques d'infraction restreinte", déclare-t-il au début de son analyse, "proviennent du répertoire de la résistance non-violente qui a été testé et enrichi ces dernières années par le mouvement américain des droits civiques. Face à un dispositif gouvernemental bureaucratisé et du fait d'un espace publicitaire exerçant une influence commerciale sur les masses, ces techniques acquièrent une nouvelle importance; elles pénètrent dans les niches d'un système inattaquable de façon frontale ; les retombées qu'elles engendrent sont sans commune mesure avec les efforts déployés, qui sont relativement restreints, car elles sont dirigées sur les failles de réseaux de communication complexes, et donc fragiles. $»^{12}$ 
Les formes inhabituelles de contestation auraient, certes, conduit d'un côté à « de violentes réactions d'auto-défense" mais, d'autre part également, «à un choc salutaire " poussant à réfléchir à la routine et aux phénomènes de refoulement routiniers. D'un point de vue psychologique, ces techniques de manifestation relèveraient de "formes ritualisées du chantage et de provocation exercées par de jeunes gens vis-à-vis de parents négligents, mais relativement indulgents ».

Ces formes de protestation ne pourraient produire un effet qu'à condition d'être mises en place de manière réfléchie, et non pas infantile. "Si l'on conçoit les nouvelles techniques comme des techniques en principe non-violentes, à portée symbolique et applicables par une tranche d'âge spécifique », résume Habermas, « dans ce cas, il n'y a aucun doute à avoir quant à leur fonction. Elles sont parfaitement adéquates (mais c'est leur seule utilité) pour faire tomber les barrières de la publicité et enclencher des processus d'éducation, de massifs processus d'éducation au sens d'Aufklärung. Les nouvelles techniques de manifestation touchent le seul point faible de ce système politique en quête de légitimation : la dépolitisation régnant au sein de vastes couches de la population. $»^{13}$

21 Dans le cadre de sa critique, cet énoncé remplissait une double fonction. D'une part, il visait à délimiter la marge de manœuvre des actions extraparlementaires que le sociologue, s'il ne les assumait pas directement, était du moins tout à fait prêt à soutenir par des arguments, et d'autre part, sa déclaration était supposée indiquer une piste permettant de s'inspirer de l'efficacité nullement négligeable du mouvement étudiant.

La devise se résumait ainsi : toucher aux points faibles de la légitimation et déclencher, par les effets publicitaires des actions non violente, ces processus d'éducation qui contribueraient potentiellement à une politisation en profondeur de la population. Pour Habermas, c'est en cela que résidait la fonction positive des sit-in, occupations de locaux et autres techniques, qu'il ne nommait pas expressément, qui trouvaient leur origine dans l'arsenal du mouvement américain des droits civiques.

23 IV. Ce ne fut certainement pas un hasard si le vade-mecum de la résistance nonviolente connu dans le monde entier, le pamphlet de Henry David Thoreau On the Duty of Civil Disobedience ${ }^{14}$, ouvrage classique de la littérature anarcho-libertaire, ne put paraître que très tardivement dans sa traduction allemande. Et ce fut sûrement tout aussi peu un hasard si sa première publication, datant de 1966, fut imprimée dans une presse artisanale de Francfort, aux éditions Galerie Patio. Il fallut attendre l'année suivante pour que paraisse chez l'éditeur zurichois Diogenes une version titrée Über die Pflicht zum Ungehorsam gegen den Staat ${ }^{15}$ qui, après de multiples rééditions, devint la version germanophone standard.

Aux États-Unis en revanche, le bréviaire de Thoreau est devenu une, si ce n'est la pierre angulaire des traditions de la résistance non-violente. Il n'est pas un débat sérieux autour des principes de la désobéissance civile qui ne se soit pas référé à ce mince ouvrage rédigé dans un style polémique. La première impulsion qui fut donnée à ces réflexions profondément critiques à l'égard du gouvernement remonte à juillet 1846 . Thoreau dut passer une nuit en prison à Concord (New Hampshire), sa ville natale, pour avoir refusé de payer ses impôts en signe de protestation contre l'esclavage encore en vigueur à l'époque et contre la guerre de conquête du Mexique subitement lancée par son pays. Un an et demi plus tard, cette expérience le conduira à présenter les 
impératifs qui ont motivé son boycott des impôts lors d'une conférence qu'il tiendra dans un lycée.

Dès sa première phrase, il déclare sans ambiguïté être animé par une défiance pratiquement sans borne à l'égard de tout gouvernement - et ce, quelle que soit la capacité de légitimation démocratique de celui-ci. Le meilleur gouvernement pour lui est celui «qui gouverne le moins», voire celui «qui ne gouverne pas du tout». Selon lui, un gouvernement peut être " au mieux un instrument utile »- mais en aucun cas davantage. La clef de voûte de sa conception résolument individualiste, c'est la conscience. Ne pouvant résoudre sa conscience à accepter une organisation politique, quelle qu'elle soit, qui s'accroche à l'esclavage, il se sent appelé à la rébellion. Un gouvernement qui est "en même temps un gouvernement d'esclaves" ne peut être, d'après les convictions de Thoreau, celui d'un citoyen libre. On mesure son rejet catégorique dans l'appel qu'il lance à ses concitoyens: "Ce peuple doit cesser de maintenir l'esclavage et de porter la guerre au Mexique, même au prix de son existence nationale $»^{16}$. Il n'a que mépris pour les décisions prises à la majorité. Les élections sont pour lui « une sorte de jeu ». Et l'électeur lui-même n'a « rien à y gagner ». Car même si l'on a voté pour ce qui est juste, cela ne veut pas dire pour autant qu'on fera ensuite quelque chose dans cette direction. Il existe indubitablement des "lois injustes", dit Thoreau. Si une loi est constituée de telle manière qu'elle fait nécessairement « de nous l'instrument de l'injustice envers notre prochain ", alors il faut l'enfreindre. L'État ne dispose ni d'un esprit supérieur ni d'une dignité supérieure qui pourraient justifier sa puissance, il ne dispose que de « la supériorité physique».

Selon Thoreau, une minorité reste impuissante tant qu'elle se conforme à la majorité ; mais elle devient irrésistible lorsqu'elle fait obstruction de tout son poids. Il affirme : «si un millier, si une centaine, si une dizaine d'hommes que je pourrais nommer - si seulement dix honnêtes gens - que dis-je? Si un seul honnête homme cessait dans notre État du Massachusetts de garder des esclaves, venait vraiment à se retirer de cette confrérie, quitte à se faire jeter dans la prison du Comté, cela signifierait l'abolition de l'esclavage en Amérique. Car peu importe qu'un début soit modeste : ce qui est bien fait au départ est fait pour toujours. $»^{17}$

27 Après avoir admis que le passage de la monarchie à la démocratie, parce que cette dernière témoigne à l'individu une plus forte considération, a véritablement marqué un progrès, Thoreau soulève finalement la question de savoir si la démocratie est vraiment " la forme de gouvernement la plus aboutie " et s'il ne serait pas possible de franchir une nouvelle étape vers la reconnaissance des droits de l'Homme. Ainsi Thoreau conclut-il son credo : «Jamais il n'y aura d'État vraiment libre et éclairé, tant que l'État n'en viendra pas à reconnaître à l'individu un pouvoir supérieur et indépendant d'où découlerait tout le pouvoir et l'autorité d'un gouvernement prêt à traiter l'individu en conséquence. $»^{18}$

On comprend évidemment pourquoi le manifeste de Thoreau a trouvé un si grand écho auprès de Martin Luther King et des autres représentants du mouvement pour les droits civiques des Noirs. Comme il s'est appuyé sur l'injustice qu'est l'esclavage pour argumenter son attaque contre l'État et le gouvernement, il pouvait sur ce point s'en référer à la constitution américaine. Somme toute, la détention d'esclaves était en contradiction avec les droits élémentaires de l'homme et du citoyen tels qu'ils étaient inscrits dans la Déclaration des Droits des États fédéraux américains. En ce sens, il a argumenté en s'appuyant sur la constitution. Il en allait autrement pour le second grief 
qu'il formula : le rejet de la guerre d'annexion du Mexique. Dans ce cas-ci, il n'a pu faire valoir aucun principe constitutionnel, mais uniquement la conviction selon laquelle une guerre qui ne sert pas la défense du pays serait contraire au droit.

Bien que l'on ait souvent pointé du doigt la partialité, l'outrance et les faiblesses dans l'argumentation de Thoreau, on ne peut lui ôter le mérite d'avoir appeler à la désobéissance face à des lois injustes et d'avoir proposé un correctif de l'État toutpuissant face à l'individu.

30 C'est peut-être chez Mahatma Gandhi que les idées de Thoreau ont eu le plus fort impact ${ }^{19}$ : déjà en Afrique du Sud il avait lu ses écrits et l'on raconte qu'il a presque toujours conservé sur lui le traité sur la désobéissance civile lors de ses fréquents séjours en prison. En menant la résistance contre la puissance coloniale britannique au moyen de marches de protestation, de boycotts des impôts et de refus d'obtempérer, il a montré comment relier les idées volontaires d'un yankee convaincu aux traditions indiennes ancestrales de renoncement à la violence. ${ }^{20}$

31 V. C'est dans les années 1960 aux États-Unis que le débat sur les principes de désobéissance civile s'est ouvert et qu'il s'est le plus largement diffusé. À partir de cette époque, il n'a plus été seulement conduit par des activistes et des auteurs proches de ce mouvement, mais également par un nombre de plus en plus important d'intellectuels renommés issus des sciences sociales, politiques et juridiques et travaillant pour les grandes universités ${ }^{21}$. Le mouvement pour les droits civiques donnait l'impression de sombrer peu à peu dans une crise d'identité, tandis que l'ensemble du pays, face à la sérieuse aggravation du conflit au Vietnam, voyait s'accroître l'ampleur du mouvement anti-guerre tout en enregistrant une multitude de désertions - dans ce contexte se posait alors la question de savoir si les méthodes de la désobéissance civile étaient conciliables avec la constitution américaine et, si oui, où se trouvaient leurs limites.

Parmi tous les grands penseurs qui ont accueilli de façon constructive l'impulsion lancée par les défenseurs des droits du citoyen, l'une des voix les plus remarquables est celle de la théoricienne du totalitarismeHannah Arendt. Dans son essai "Civil Disobedience " rédigé à partir d'une conférence prononcée le $1^{\mathrm{er}}$ mai 1970 lors du symposium "La loi est-elle morte?", elle a fourni un plaidoyer en faveur d'une institutionnalisation de la désobéissance civile sous une forme bien définie ${ }^{22}$. Tout d'abord, elle part du constat que la désobéissance partielle à l'égard du système juridique est devenu un phénomène de masse non seulement aux États-Unis, mais aussi dans de nombreux endroits au monde. D'après elle, ceci serait déjà un indice infaillible révélant le déclin de l'autorité - indice que l'on considérerait peut-être un jour comme "l'événement éminent" des années 1960. Il serait même possible de voir dans le processus d'effritement de l'autorité gouvernementale et dans les tendances qu'a le système politique à se désintégrer les symptômes à l'origine de révolutions futures. Dans cette perspective, il ne serait pas erroné de voir dans les réflexions d'Arendt sa contribution à la défense, si ce n'est à la sauvegarde, de la démocratie libérale.

Pour mettre en relief l'attitude fondamentale adoptée par la personne qui refuse d'obéir tout en restant dans le cadre de la constitution, elle délimite celle-ci à l'aide de deux figures: d'un côté, le criminel; de l'autre, le révolutionnaire. Selon elle, la différence principale par rapport au criminel est que la personne qui refuse d'obéir enfreint la justice non pas dans l'ombre, mais en public; de plus, elle n'agit pas exclusivement dans son propre intérêt, mais « au nom d'un groupe et pour celui-ci ». Quant au révolutionnaire, c'est dans le principe de non-violence qu'elle voit la 
différence majeure. Bien qu'ils exigent tout autant du changement, les adeptes de la désobéissance civile acceptent le «cadre de l'autorité établie et la légitimité générale $\mathrm{du}$ système juridique». Pour Arendt, l'immanence politique ainsi que juridique représente l'élément central dans la façon d'évaluer la désobéissance civile.

Elle va même jusqu'à déclarer le phénomène, dans ses origines aussi bien que dans son contenu, comme étant " proprement américain ». À la différence de tous les autres pays où l'on pratique de telles formes de résistance, la république américaine serait le seul État à pouvoir admettre et intégrer ces pratiques - si ce n'est au sens littéral, du moins " en harmonie avec l'esprit de ses lois». Ceci serait lié au fait que la Révolution américaine est issu de l'expérience vécue par les colons, et non d'une théorie. Étant donné que le concept de droit n'aurait pas été entièrement défini, il y aurait éventuellement la possibilité d'aller jusqu'à ancrer le principe de désobéissance civile dans la constitution américaine.

Vouloir justifier légalement une atteinte au droit s'avérant être un paradoxe manifeste, il n'y aurait cependant aucune possibilité d'établir directement la désobéissance civile en tant que droit positif. Pour Arendt, la seule issue possible serait une institutionnalisation politique de la résistance exercée par des minorités agissant de manière non-violente. Un premier pas effectué dans cette direction consisterait alors à garantir à de tels groupuscules la même reconnaissance que celle tout naturellement accordée à d'autres groupes d'intérêt évoluant aux États-Unis.

VI. Dans le domaine de la théorie du droit, la conception des problèmes liés à la désobéissance civile qui eut le plus d'influence nous vient du spécialiste américain de philosophie morale John Rawls. Bien qu'il soit profondément sceptique quant à la possibilité de fixer des règles juridiques définissant plus précisément la pratique de telles formes de résistance, il a néanmoins formulé en 1971 dans sa "Theory of Justice $»^{23}$ quelques principes fondamentaux d'actions non-violentes.

37 Pour ce faire, il commence par poser un cadre restrictif consistant à déclarer que toute théorie sur la désobéissance civile ne peut être envisagée qu'en tant que "cas particulier dans une société presque juste». Puisque les formes de résistance nonviolente sont inaptes à changer ou à éliminer un régime dictatorial, une telle théorie ne peut faire autorité pour les formes de gouvernement en général. Elle suppose d'une part une démocratie en état de marche et d'autre part la reconnaissance de la constitution par les citoyens, y compris donc par les adeptes de la désobéissance. Au fond, le problème de la désobéissance civile est pour lui un "conflit de devoirs »conflit explicite face au pouvoir exécutif, et implicite face au législatif. La question décisive est : à partir de quel moment l'obligation de se plier aux lois promulguées par la majorité législative ou à l'action gouvernementale que cette majorité soutient, peutelle être abandonnée au profit du droit à défendre ses libertés ou du devoir de résistance. Comme une telle question interroge finalement la légitimité des règles définies par la majorité, ce problème doit être considéré comme le "test crucial pour toute théorie sur le fondement moral de la démocratie ». La résistance non-violente s'adresse ainsi au « sens de la justice de la majorité de la société ».

D’après sa définition, la désobéissance civile s'exprime dans « un acte public, nonviolent, décidé en conscience, mais politiquement contraire à la loi et accompli le plus souvent dans le but de mener à un changement dans les lois ou la politique du gouvernement $\aleph^{24}$. Par des moyens contraires à la loi mais non-violents, une minorité cherche à pousser une majorité à examiner son action ou celle du gouvernement pour 
déterminer si elle concorde encore avec les principes de justice qui sous-tendent la constitution et les institutions de la société.

La définition de Rawls culmine dans la formulation paradoxale selon laquelle la désobéissance civile est une « désobéissance à la loi qui reste dans le cadre de la fidélité à la loi $»^{25}$. Même si la loi est enfreinte d'un point de vue formel, le caractère public et non-violent de l'acte de résistance exprime toutefois sa bonne volonté à se soumettre sans résister aux conséquences juridiques. Cette acceptation est d'après Rawls un indice révélant à la fois la fidélité des acteurs aux lois, la conscience qui est au fondement de leurs actes, ainsi que le sérieux et la bonne foi exprimés précisément à travers leurs actes. C'est cette caractéristique de « désobéissance à la loi qui reste dans le cadre de la fidélité à la loi » qui dégage de façon nette l'immanence systémique des objectifs et des moyens déployés par les adeptes de la désobéissance.

VII. Les travaux de Rawls ont rencontré une réception particulièrement favorable en République fédérale au début des années 1980, lorsque le mouvement de protestation qui s'opposait à la décision de l'OTAN d'augmenter le potentiel militaire atteignit son point culminant. Dans les débats de l'époque ${ }^{26}$, on retrouve une fois encore Jürgen Habermas qui, dans une approche théorique du droit, énonça des principes de désobéissance qui se réfèrent aux grandes lignes tracées par Rawls.

"La désobéissance civile", résume Habermas dans un examen élargi de la différenciation, « est une protestation fondée moralement qui ne doit pas uniquement reposer sur des convictions de foi ou des intérêts personnels ;

- c'est un acte public qui, en règle générale, est annoncé à l'avance et dont le déroulement peut être prévu par la police ;

- elle inclut la violation préméditée de normes juridiques particulières, sans affecter l'obéissance à l'ordre juridique dans son ensemble ;

- elle exige la disponibilité à répondre des conséquences juridiques de la violation de la norme ; L'infraction au règlement par laquelle se manifeste la désobéissance civile a exclusivement un caractère symbolique - ce qui implique de s'en tenir à des moyens nonviolents de protestation ${ }^{27}$. Cette définition contient la formulation la plus aboutie des critères qui doivent permettre d'évaluer une action de résistance au titre de désobéissance civile.

Habermas associe la réception de cette représentation américaine de la protestation et, d'une certaine manière, de la constitution, à l'espoir qu'un jour la désobéissance civile puisse faire son entrée aussi en Allemagne « en tant qu'élément symptomatique d'une culture politique arrivée à maturité ». Bien que ses méthodes évoluent en marge de la fidélité à la loi, elles s'inscrivent dans la normalité d'une démocratie fondée sur le droit. Il voit dans l'acceptation de telles formes de résistance non-violentes une "pierre de touche servant à reconnaître le stade de maturité de la première république démocratique instaurée sur le sol allemand», un «test pour l'État de droit démocratique » dans son ensemble.

Quoiqu'il y ait assez de points de vue appuyant le scepticisme d'Hannah Arendt en ce qui concerne la transférabilité de la tradition de désobéissance civile sur d'autres systèmes constitutionnels, les actions non-violentes, en particulier celles qui ont été entreprises dans le cadre du mouvement ouest-allemand pour l'écologie et pour la paix - comme par exemple les sit-in de Mutlangen au début des années 1980 - ont montré que la capacité à pratiquer de telles formes de résistance de manière responsable, ainsi que l'acceptation de tels comportements, impossibles à concilier avec des conceptions 
autoritaires, se sont développées dans la sphère publique ${ }^{28}$. En ce sens, en Allemagne aussi, un élément crucial de la culture de protestation américaine est désormais considéré comme plus naturel.

Conclusion. La protestation est un concept positionnel. Elle s'élève contre un dysfonctionnement qu'elle démasque, elle porte une accusation, rend public, s'adresse à des partenaires pour lier des alliances, propose des solutions et essaie de mettre en place des démarches pour leur application pratique.

La protestation en soi n'a aucun sens. Elle ne prend pas forme dans un espace vide. Ce n'est qu'en relation avec quelque chose, dans une attitude critique, oppositionnelle ou adverse vis-à-vis d'un détenteur ou d'une instance de pouvoir qu'elle prend sens. Par la protestation s'établit une relation avec un ou plusieurs interlocuteurs auxquels s'adressent l'appel, la revendication, et parfois également une menace. Une personne, une institution ou une organisation entre ainsi en jeu, en tant qu'élément du système politique où les individus qui lancent leur appel et ceux qui agissent se répondent mutuellement. Et dans cette constellation, il est question de problèmes, de lacunes, de paradoxes, de foyers de crise et de danger.

En ce sens, la protestation est toujours intégrée au système dans lequel elle se manifeste. Elle est intégrée de manière négative lorsqu'elle s'oppose à une lacune ou à un dysfonctionnement; elle est intégrée de manière positive lorsqu'elle vise la correction, la résorption ou le changement de ces défauts.

La résistance est à comprendre comme un concept positionnel dans un sens encore plus marqué. Les formes qu'elle revêt peuvent être très variées. Elles vont du refus d'obéissance à la perpétration d'actes révolutionnaires, en passant par l'opposition, la rébellion et l'obstruction.

Le concept ne se distingue ni par des critères de légalité, ni par des critères de légitimité. Son rapport à l'ordre social et politique établi dépend de l'endroit où l'on fait passer la frontière entre résistance passive et active, légale et illégale, légitime et illégitime, ce qui en rend la définition difficile. Implicitement, la question soulevée est celle de l'appréciation morale, juridique et politique.

Le concept de résistance ne fait pas qu'élargir le terrain de la protestation : il franchit également une double frontière - dans une perspective pratique ainsi que légitimatrice. Tandis que la protestation peut se référer à une marge de manœuvre admise comme légale, la résistance ne se cantonne pas forcément à ce cadre.

Malgré tous ces impondérables, le droit à la résistance relève communément depuis l'Antiquité d'une culture du droit. Dans l'article 20, paragraphe 4, la loi fondamentale allemande garantit elle-même, dans un sens très précisément défini, un droit de résistance. Ce n'est qu'à posteriori que celui-ci a été ajouté - en mai 1968 - et, en tout état de cause, à des fins purement défensives. Son rôle consiste à pouvoir en faire usage dans des cas exceptionnels afin de défendre, voire de sauver l'ordre constitutionnel. Ce droit fait écho aux expériences négatives qui ont été faites avec l'effondrement de la République de Weimar.

51 Dans le concept de résistance passive, soit de désobéissance civile, s'établit un lien entre forme de protestation et forme de résistance. Tout en ayant conscience de son ambiguïté et du danger inhérent qui consiste à n'y voir qu'un élément transitoire sur le chemin d'une déstabilisation de l'ordre constitutionnel, Habermas a délimité cette infraction aux règles en s'appuyant sur sa forme symbolique et non-violente. 
Pour une telle politique de l'infraction restreinte, les critères suivants sont déterminants :

- sa légitimité morale,

- le caractère public de ses options d'action,

- la violation consciente de normes juridiques particulières,

- la disponibilité à répondre également des conséquences juridiques de ces normes,

- le caractère exclusivement symbolique de cet acte.

Tout ce qui s'étend au-delà de ce périmètre n'est pas envisagé. Le point-clé est l'idée de civilité dans l'acte de désobéissance en tant que forme de résistance, la seule qui soit justifiable dans le contexte d'un État constitutionnel démocratique.

\section{NOTES}

1. En allemand, le mot « Protest » signifie à la fois « contestation » et « protestation ». (N.d.E.)

2. Ulrike Meinhof, «Vom Protest zum Widerstand » (« De la protestation à la résistance »), in : Konkret 5/1968, p. 5.

3. Ibid, p. 139.

4. Ibid. p. 140.

5. C'était notamment l'objectif déclaré de membres de la Kommune I comme Dieter Kunzelmann. Celui-ci avait ainsi formulé son credo sur une carte postale : «L'aventure la plus visible de tout être humain consiste à accomplir une série d'actes qui enfreignent la loi... » Extrait de : Frank Böckelmann, Herbert Nagel (dir.), Subversive Aktion - Der Sinn der Organisation ist ihr Scheitern, Francfort/Main, 1976.

6. Peter Schneider, "Wir haben Fehler gemacht», in: Ansprachen: Reden - Notizen - Gedichte, Berlin-Ouest 1970, p. 12-14.

7. «Über den Begriff der politischen Beteiligung ». Introduction à l'étude empirique menée par l'Institut de recherche sociale de Francfort : Jürgen Habermas, Ludwig von Friedeburg, Christoph Oehler, Friedrich Weltz, Student und Politik - Eine soziologische Untersuchung zum politischen Bewußtsein Frankfurter Studenten, Neuwied/Berlin, 1961, p. 11-55.

8. Jürgen Habermas, L'espace public - Archéologie de la publicité comme dimension constitutive de la société bourgeoise, Paris, 1978.

9. Wolfgang Abendroth (et al.), Die Linke antwortet Jürgen Habermas, Francfort/Main, 1968.

10. Voir : Uwe Bergmann (dir.), Bedingungen und Organisation des Widerstandes - Der Kongress in Hannover, Berlin-Ouest, 1967, p. 100-102.

11. Jürgen Habermas, «Die Scheinrevolution und ihre Kinder - Sechs Thesen über Taktik, Ziele und Situationsanalysen der oppositionellen Jugend », in: Frankfurter Rundschau, 5 juin 1968.

12. Jürgen Habermas, «Die Scheinrevolution und ihre Kinder », in: ibid., Protestbewegung und Hochschulreform, Francfort/Main 1969, p. 191.

13. Ibid., p. 192.

14. Henry David Thoreau, Resistance to Civil Government, in: Aesthetic Papers, éd. Elizabeth Peabody, Vol. 1, $\mathrm{N}^{\circ} 1$, Boston 1849 ; diffusé ensuite le plus souvent sous le titre de Civil Disobedience ou encore On the Duty of Civil Disobedience.

15. Henry David Thoreau, Über die Pflicht zum Ungehorsam gegen den Staat, Zurich, 1967. 
16. Ibid., p. 24. Traduction française par Micheline Flak, Christine Demorel et Laurence Vernet, Éditions Climats, coll. « L'éclipse » n 4, Castelnau-le-Lez, 1992.

17. Ibid., p. 47.

18. Ibid., p. 96.

19. Cf. Mahatma Gandhi, Wohlfahrt für alle, dir. B. Kumarappa, Bellnhausen, 1962.

20. On notera également la thèse originale d'une «américanisation de Gandhi » d'après laquelle le pacifisme radical aux États-Unis serait dû à une adaptation des idées de Gandhi ; cf. le chapitre «Radical Pacifism, The Americanization of Gandhi » in : Maurice Isserman, If I Had a Hammer... The Death of the Old Left and the Birth of the New Left, New York, 1987, p. 125-169.

21. Voir : Hugo Adam Bedau (dir.), Civil Disobedience Theory and Practice, New York 1969 ; Archibald Cox/Mark Howe/J.R. Wiggins, Civil Rights, the Constitution and the Courts, Cambridge, Mass., 1967 ; Robert T. Hall, The Morality of Civil Disobedience, New York, 1971; Daniel B. Stevick, Civil Disobedience and the Christian, New York, 1969 ; Michael Walzer, Obligations, Cambridge, Mass., 1970 ; Charles E. Whittaker/William Sloane Coffin Jr., Law, Order and Civil Disobedience, Washington, D.C. 1967 ; Howard Zinn, Disobedience and Democracy, New York, 1968.

22. Hannah Arendt, «Ziviler Ungehorsam », in : ibid., Zur Zeit - Politische Essays, Marie Luise Knott (dir.), Berlin-Ouest, 1986, p. 119-159.

23. Version française : Théorie de la justice, trad. Catherine Audard, Paris, 1987.

24. Ibid., p. 411.

25. Ibid.

26. Cf. Peter Glotz (dir.), Ziviler Ungehorsam im Rechtsstaat, Francfort/Main, 1983.

27. Jürgen Habermas, «Ziviler Ungehorsam - Testfall für den demokratischen Rechtsstaat », in : Die neue Unübersichtlichkeit, Francfort/Main, 1985, p. 83.

28. Cf. Gernot Jochheim, Die gewaltfreie Aktion - Idee und Methoden, Vorbilder und Wirkungen, Hambourg, 1984 ; Thomas Laker, Ziviler Ungehorsam, Baden-Baden, 1986.

\section{RÉSUMÉS}

Cet article démontre qu'un type de contestation non violente s'est acclimatée en République fédérale d'Allemagne entre le mouvement des étudiants de 1968 et les mouvements pacifiques des années 1980. L'auteur analyse les conceptions développées par Peter Schneider, Jürgen Habermas, Henry David Thoreau, Hannah Arendt et John Rawls afin de définir les concepts d' "infraction restreinte », de " protestation », « désobéissance civile », « résistance » et leur réception en Allemagne.

This paper aims at showing that a certain type of non-violent protest emerged in the Federal Republic of Germany between the 1968 student movement and the peace movement of the 1980's. The author looks into the work of Peter Schneider, Henry David Thoreau, Hannah Arendt and John Rawls in order to define the concepts of "restricted infringement", "protest", "civil disobedience" and "resistance" as well as their reception in Germany. 
INDEX

Keywords : protest, resistance, civil disobedience, democracy, civil rights movement

Mots-clés : protestation, résistance, désobéissance civile, démocratie, mouvements pour les droits civiques

\section{AUTEURS}

\section{WOLFGANG KRAUSHAAR}

Hamburger Institut für Sozialforschung, Allemagne

his@his-online.de 